\title{
Editorial
}

\section{Combined choroid plexus coagulation and endoscopic third ventriculostomy: is North America ready?}

Mark S. Souweidane, M.D.

\footnotetext{
Neurological Surgery and Pediatrics, Weill-Cornell Medical College, New York, New York
}

Any advance made in the therapeutic approach toward CSF diversion in infants, especially those afflicted with posthemorrhagic hydrocephalus of prematurity, is a welcome addition. Choroid plexus ablation has recently resurfaced as a beneficial adjunct in this quest. ${ }^{3,4,7}$ Specifically, combining choroid plexus cauterization (CPC) with endoscopic third ventriculostomy (ETV) has been espoused as superior to shunt placement or ETV alone. ${ }^{8}$ Much of the evidence to support superiority is born out of Dr. Benjamin Warf's intensive and noble work in East Africa. Although the results of that experience have been carefully analyzed, inherent limits exist in extrapolating that experience to children in developed countries due to inherent and uncontrollable population differences. The level of neonatal and perioperative care, threshold for seeking medical care, lifelong risk of shunt dependency, imaging capabilities, infectious disease practices, nutritional status, cultural beliefs, and different shunt device designs are several differences that obscure accurate comparative assessments when using clinical case series from sub-Saharan Africa. In short, the work of Dr. Warf is persuasive but deserves validation in developed countries prior to universal integration into practice. Other than a recent, single-institution, and retrospective evaluation in infants with posthemorrhagic hydrocephalus of prematurity, no other comparative evaluation originating in North America is available for corroboration. ${ }^{1}$

In this issue of Journal of Neurosurgery: Pediatrics Dr. Kulkarni and colleagues, through the Hydrocephalus Clinical Research Network (HCRN), report on their initial experience from North America evaluating combined ETV + CPC in the management of infants with hydrocephalus. ${ }^{6}$ A review of a multiinstitutional prospective registry was conducted for hydrocephalic children (newly diagnosed and with failed CSF shunt or ETV) who were 2 years of age or less and treated with ETV + CPC. Outcome and morbidity were retrospectively evaluated and compared with data obtained from the HCRN Core Data Project registry for age-matched infants undergoing firsttime CSF shunt insertion. Time to first treatment failure was the primary outcome.

The results indicate that ETV $+\mathrm{CPC}$ is a reasonably useful and relatively safe alternative to shunt placement as up-front therapy for CSF diversion in young children $(<2$ years). Treatment in half of all patients failed, with a median time to failure of 30 days. Univariate analysis failed to reveal any predictor of success including age, etiology, and degree of choroid plexus coagulation. Importantly, only 2 patients $(6 \%)$ had a postinfectious etiology and only $11(31 \%)$ had fast imaging employing steady-state acquisition/constructive interference in steady state (FIESTA/ CISS) MRI sequences performed preoperatively, making meaningful evaluation of these variables problematic. Two patients $(6 \%)$ developed postoperative CNS infections. Although seemingly unrelated, having 3 deaths $(17 \%)$ in 18 patients treated with ETV + CPC seems excessive for this population (a mortality rate in the control cohort is not reported). When compared with a control group of children treated with CSF shunt as a primary procedure, the survival rate of ETV + CPC was lower throughout the 1st year of follow-up.

Methodological concerns are responsibly pointed out by the authors, and most notably include the lack of any independent adjudication of outcome. One cannot therefore exclude a potential for bias in the decision to offer subsequent CSF diversion. The children treated with ETV + CPC in general were older compared with their counterparts undergoing CSF shunt insertion ( $45 \%$ vs $24 \%>6$ months). This mismatch might artificially increase the effectiveness of the ETV + CPC given that age is inversely related to treatment failure for all forms of hydrocephalus treatment. It is rewarding to learn that the HCRN is planning a prospective study with independent observation of outcome.

Missing from this work is a comprehensive appraisal of the eventual outcome of children in whom initial ETV + CPC failed $(n=18)$. It is plausible that ETV + CPC might predispose a child receiving a CSF shunt to a complication profile greater than if shunt placement had been done up front. The potential for increased incidence of shunt infection following a previous CSF diversionary procedure is more than hypothetical. ${ }^{5}$ Additionally, nothing is known regarding the CSF composition after extensive CPC. Potential alterations in CSF protein or cellular composition might occur and might also impart a higher 
rate of catheter obstruction in the child receiving ETV + CPC compared with those undergoing a first-time CSF shunt insertion. This concern is somewhat tempered by a recent publication addressing these issues, but again extrapolating that experience to a North American population might not be applicable. ${ }^{9}$ In other words, time to first procedure failure might not be the most meaningful measure of comparative effectiveness. Malfunction rates for the group of "failed" children receiving shunts after unsuccessful ETV + CPC therefore would be a useful measure to include in the risk assessment.

Outcome included an assessment of "complications or new neurological deficits," admittedly a good measure of surgical morbidity but possibly not as good for eventual performance. Making the assumption that the degree of cerebral injury is roughly equivalent between a flexible fiberscope and a ventricular catheter might be acceptable based solely on relative device dimensions. The potential for injury between the techniques extends further, however, in that CPC includes a theoretical risk of thermal injury to structures closely approximating the choroid plexus, notably the bilateral thalami and fornices. ${ }^{2}$

The inability to monitor subtler developmental or cognitive outcome is certainly understandable using the current HCRN registry, the young age, and the relatively short follow-up. This shortcoming is by no means unique to this particular clinical study, as meaningful long-term functional outcome assessments are nearly universally absent from most contemporary clinical studies comparing different methods of CSF diversion. It is not known if these measures are included in the prospective HCRN data collection, but one would hope that this collaborative group makes every attempt to incorporate validated developmental outcomes for young children.

The authors have provided needed information for further consideration of ETV + CPC as a valid up-front therapeutic alternative to CSF shunt placement for infants with hydrocephalus. What they have not yet demonstrated is that ETV + CPC is any better than ETV alone. Regrettably there was no contemporaneous control group of infants being treated with ETV alone. In fact, when the ETV Success Score was used as an estimate of relative effectiveness, the predicted and actual success rates at 6 months were roughly equivalent (50\% vs $52 \%)$. It thus remains undecided if the described procedure provides a benefit over ETV alone in this population.

The HCRN is to be congratulated for testing a legitimate hypothesis in a representative North American population. Their results support the consideration of using ETV + CPC as a therapeutic option in infants with hydrocephalus. Choosing this treatment approach, however, exposes the child to a somewhat higher failure rate compared with shunt placement. This differential outcome may be acceptable given the relative lifelong burden of CSF shunts. Importantly, it was not shown that ETV + $\mathrm{CPC}$ is any better than ETV alone in this clinical scenario. Further clarification of these issues is anticipated through the HCRN and other groups that can use sound clinical study methods and a commitment to advance the care of this challenging patient population.

(http://thejns.org/doi/abs/10.3171/2014.4.PEDS1450)

\section{Disclosure}

Dr. Souweidane is a consultant for Aesculap.

\section{References}

1. Chamiraju P, Bhatia S, Sandberg DI, Ragheb J: Endoscopic third ventriculostomy and choroid plexus cauterization in posthemorrhagic hydrocephalus of prematurity. Clinical article. J Neurosurg Pediatr 13:433-439, 2014

2. Elliot-Lewis E, Mason AM, Barrow DL: Evaluation of a new bipolar coagulation forceps in a thermal damage assessment. Neurosurgery 65:1182-1187, 2009

3. Griffith HB: Endoneurosurgery: endoscopic intracranial surgery. Adv Tech Stand Neurosurg 14:2-24, 1986

4. Griffith HB, Jamjoom AB: The treatment of childhood hydrocephalus by choroid plexus coagulation and artificial cerebrospinal fluid perfusion. Br J Neurosur 4:95-100, 1990

5. Kestle JRW, Riva-Cambrin J, Wellons JC III, Kulkarni AV, Whitehead WE, Walker ML, et al: A standardized protocol to reduce cerebrospinal fluid shunt infection: the Hydrocephalus Clinical Research Network Quality Improvement Initiative. Clinical article. J Neurosurg Pediatr 8:22-29, 2011

6. Kulkarni AV, Riva-Cambrin J, Browd SR, Drake JM, Holubkov R, Kestle JRW, et al: Endoscopic third ventriculostomy and choroid plexus cauterization in infants with hydrocephalus: a retrospective Hydrocephalus Clinical Research Network study. Clinical article. J Neurosurg Pediatr [epub ahead of print July 4, 2014. DOI: 10.3171/2014.6.PEDS13492]

7. Pople IK, Ettles D: The role of endoscopic choroid plexus coagulation in the management of hydrocephalus. Neurosurgery 36:698-702, 1995

8. Warf B: Comparison of endoscopic third ventriculostomy alone and combined with choroid plexus cauterization in infants younger than 1 year of age: a prospective study in 550 African children. J Neurosurg 103 (6 Suppl):475-481, 2005

9. Warf BC, Bhai S, Kulkarni AV, Mugamba J: Shunt survival after failed endoscopic treatment of hydrocephalus. Clinical article. J Neurosurg Pediatr 10:463-470, 2012

\section{Response}

Abhaya V. Kulkarni, M.D., Ph.D.,
fOr the Hydrocephalus Clinical Research Network

Hospital for Sick Children, University of Toronto, Ontario, Canada

We thank Dr. Souweidane for his thoughtful comments. We agree with him on all substantive points.

Time to first failure is not the only-or even the most-important outcome for this population, but it is the most immediate and accessible to measurement. Downstream outcomes (including the consequences of shunt insertion after failed ETV + CPC and neurocognitive effects of the procedure and the altered brain physiology) will probably prove to be more relevant to the child's ultimate quality of life. The research infrastructure of the HCRN allows us to monitor outcome in children over many years, and this should shed further light on issues of long-term complications. The HCRN is also implementing neurocognitive testing in more of our study protocols, in addition to investigating the use of early imaging, for example diffusion tensor imaging, as a surrogate measure for long-term neurocognitive outcome. However, because 


\section{Editorial}

most children will undergo multiple interventions, it will remain challenging to attribute any deleterious effects to a particular single intervention alone, for example, thermal injury.

Our study failed in providing a reasonable comparison cohort against which to answer the question: is ETV + CPC more effective than ETV alone? Although our findings were limited, we did provide indirect evidence by demonstrating improved outcome for near-complete CPC $(\geq 90 \%)$ compared to lesser degrees of CPC. The data from Warf's work, however, are very compelling in this regard, consistently showing a beneficial effect for ETV + CPC compared with ETV alone in multiple analyses across a range of hydrocephalus etiologies. ${ }^{1-3}$

We agree that we are in the very early stages of exploring the role of ETV + CPC in the North American population, with much work left to be done. The HCRN remains committed to addressing this issue, and we hope that our future prospective study will further our under- standing of where ETV + CPC should fit in the armamentarium of North American pediatric neurosurgeons.

\section{References}

1. Warf BC: Comparison of endoscopic third ventriculostomy alone and combined with choroid plexus cauterization in infants younger than 1 year of age: a prospective study in 550 African children. J Neurosurg 103 (6 Suppl):475-481, 2005

2. Warf BC, Mugamba J, Kulkarni AV: Endoscopic third ventriculostomy in the treatment of childhood hydrocephalus in Uganda: report of a scoring system that predicts success. Clinical article. J Neurosurg Pediatr 5:143-148, 2010

3. Warf BC, Tracy S, Mugamba J: Long-term outcome for endoscopic third ventriculostomy alone or in combination with choroid plexus cauterization for congenital aqueductal stenosis in African infants. Clinical article. J Neurosurg Pediatr 10:108-111, 2012

Please include this information when citing this paper: published online July 4, 2014; DOI: 10.3171/2014.4.PEDS1450. 